ESAIM: PROCEEDINGS, April 2012, Vol. 36, p. 121-125

Danièle Fournier-Prunaret, Laura Gardini, \& Ludwig Reich, Editors

\title{
SYMBOLIC DYNAMICS AND LYAPUNOV EXPONENTS FOR LOZI MAPS
}

\author{
Diogo Baptista ${ }^{1}$ And RicARdo SEverino ${ }^{2}$
}

\author{
Dedicated to José Sousa Ramos
}

\begin{abstract}
Building on the kneading theory for Lozi maps introduced by Yutaka Ishii, in 1997, we introduce a symbolic method to compute its largest Lyapunov exponent. We use this method to study the behavior of the largest Lyapunov exponent for the set of points whose forward and backward orbits remain bounded, and find the maximum value that the largest Lyapunov exponent can assume.
\end{abstract}

AMS (2000) subject classification. Primary: 37D45; Secondary: 37E30, 37E05.

Keywords. Iteration of maps, Lozi maps, Symbolic dynamics, Lyapunov exponents.

Résumé. Nous étudions ici une nouvelle méthode pour calculer le plus grand exposant de Liapounov pour la famille des applications de Lozi, en utilisant la théorie du kneading pour ces applications, introduite par Yutaka Ishii. Avec cette méthode, on trouve la valeur maximale que cet exposant de Liapounov peut avoir dans la region des paramètres sur laquelle l'attracteur de l' application est fractal.

Mots clefs. Transformation itérative, Transformation de Lozi, Dynamique symbolique, Exposant de Lyapunov.

\section{INTRODUCTION}

The identification of chaos is an important problem that can be solved by measuring the largest Lyapunov exponent of a dynamical system. Indeed, since the Lyapunov exponents quantify the divergence of initially close state-space trajectories, we can use them to estimate the amount of chaos of the dynamics. In the following, we introduce a method to compute the largest Lyapunov exponent for the family of plane maps, known as Lozi maps, from the symbolic dynamics of its attractor.

In 1978, René Lozi [5] pointed out numerically that a certain piecewise linear plane map displayed a strange attractor. Since then, we call the two-parameter family of piecewise affine homeomorphisms of the plane $\mathcal{L}_{a b}: \mathbb{R}^{2} \rightarrow \mathbb{R}^{2}$ defined by

$$
\mathcal{L}_{a b}(x, y)=(1-a|x|+y, b x), \quad a, b \in \mathbb{R}, b \neq 0,
$$

the Lozi family of maps. It was for this family of maps that Yataka Ishii [3] generalized Milnor and Thurston's kneading theory with the introduction of the appropriate symbolic dynamics, from which we are able to compute the largest Lyapunov exponent of the map.

${ }^{1}$ School of Technology and Management, Polytechnic Institute of Leiria, Leiria, CIMA-UE, University of Évora, Évora, Campus 2, Morro do Lena - Alto do Vieiro 2411-901 Leiria, Portugal; email: baptista@estg.ipleiria.pt.

2 CIMA-UE, University of Évora, Rua Romão Ramalho 59, 7000-671 Évora, Portugal; email: ricardo@math.uminho.pt.

(c) EDP Sciences, SMAI 2012 


\section{LOZI MAPS KNEADING THEORY}

Consider a Lozi map $\mathcal{L}=\mathcal{L}_{a b}$ and let us denote by $\mathbf{A}_{\mathcal{L}_{a b}}$ its attractor. In 1992, Z. Liu, H. Xie, Z. Zhu, and Q. Lu [6] showed that, for parameters values $(a, b)$ satisfying

$$
\left\{\begin{array}{l}
0<b<1 \\
a>b+1 \\
2 a>4-b
\end{array}\right.
$$

the map $\mathcal{L}_{a b}$ has a strange attractor; let us denote by $\triangle_{\mathcal{L}}$ the corresponding region of the parameter space. From now on, we assume that the parameters $(a, b)$ satisfy conditions $(2)$.

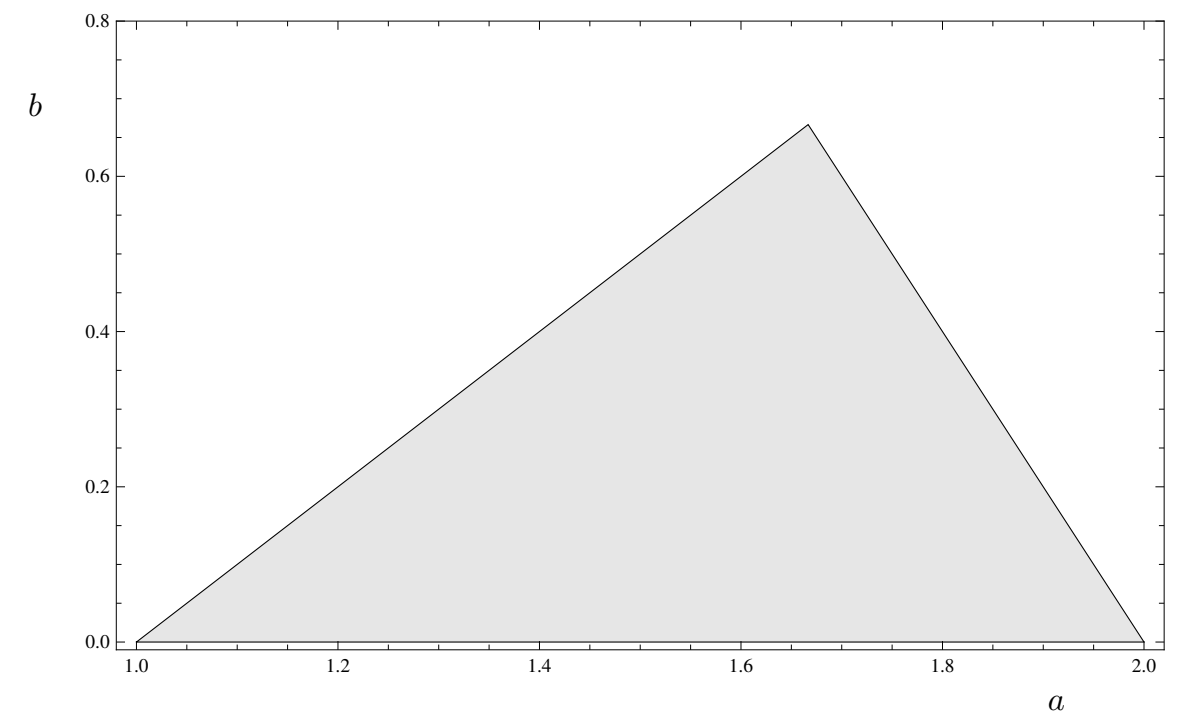

FIGURE 1. The region $\triangle_{\mathcal{L}}$ of parameter values for which the map $\mathcal{L}_{a b}$ has a strange attractor.

In 1997, Yutaka Ishii [3] developed a kneading theory for Lozi maps, as follows: to any point $X \in \mathbf{A}_{\mathcal{L}_{a b}}$, one can associate a bi-infinite symbolic sequence $\pi_{\mathcal{L}}(X)$ given by

$$
\pi_{\mathcal{L}}(X) \equiv \cdots \varepsilon_{-3} \varepsilon_{-2} \varepsilon_{-1} \cdot \varepsilon_{0} \varepsilon_{1} \varepsilon_{2} \cdots,
$$

where the symbol $\varepsilon_{i}$ is related with the $x$-relative position of the iterate $i$ of $X$ :

$$
\varepsilon_{i}= \begin{cases}-1 & \text { if } \mathcal{L}_{a b}^{i}(X)_{x}<0 \\ +1 & \text { if } \mathcal{L}_{a b}^{i}(X)_{x}>0\end{cases}
$$

denoting by $\mathcal{L}_{a b}^{i}(X)_{x}$ the $x$-component of the point $\mathcal{L}_{a b}^{i}(X)$. In his paper, Ishii used a different symbol, $*$, to code the case in which the point $\mathcal{L}_{a b}^{i}(X)$ belongs to the vertical axis, i.e., if $\mathcal{L}_{a b}^{i}(X)_{x}=0$, then $\varepsilon_{i}=*$. This new symbol plays a double role, in the sense that one can substitute it either by -1 or by +1 . This ambiguity makes $\pi_{\mathcal{L}}$ a multi-valued map and we say that any element of $\pi_{\mathcal{L}}(X)$ is an itinerary of the point $X$. The subsequence $\varepsilon_{0} \varepsilon_{1} \varepsilon_{2} \cdots$ is often called the forward itinerary of the point $X$.

As proved by Ishii, the map $\pi_{\mathcal{L}}$ conjugates the action of the Lozi map on the attractor $\mathbf{A}_{\mathcal{L}_{a b}}$ and the shift map on a subset of $\{-1,+1\}^{\mathbb{Z}}$. In what follows, we will compute the largest Lyapounov eigenvalue associated with $\mathbf{A}_{\mathcal{L}_{a b}}$ from the symbolic code of the forward orbit of the same point. 
From some simple algebraic computations, one can conclude that, if both conditions $b<a+1$ and $b>1-a$ are satisfied, then the Lozi map $\mathcal{L}_{a b}$ has two fixed points. Let us denote by $A$ the first quadrant fixed point,

$$
A=\left(\frac{1}{a+1-b}, \frac{b}{a+1-b}\right),
$$

and consider the point $I \in \mathbb{R}^{2}$ obtained from the intersection of the unstable manifold of the fixed point $A$ with the horizontal axis. An easy computation gives us its expression,

$$
I=\left(\frac{2+a+\sqrt{a^{2}+4 b}}{2(1+a-b)}, 0\right)
$$

and it is not difficult to infere that this point $I$ belongs to the attractor $\mathbf{A}_{\mathcal{L}_{a b}}$, being also its rightmost point. Next, we present our main result, which will allow us to compute the largest Lyapunov exponent associated with $\mathbf{A}_{\mathcal{L}_{a b}}$ using a forward itinerary of this point $I$.

\section{Symbolic Dynamics And Lyapunov exponents}

Lyapunov exponents describe the behavior of vectors in the tangent space of the phase space and are defined from the Jacobian matrix, $J_{a b}=J\left(\mathcal{L}_{a b}\right)$, of the map, see [7]. In this case, we have

$$
J_{a b}=\left[\begin{array}{cc}
\mp a & 1 \\
b & 0
\end{array}\right] \text {. }
$$

Given a Lozi map $\mathcal{L}_{a b}$, let $M_{a b}=M\left(\mathcal{L}_{a b}\right)$ be the matrix defined by

$$
M_{a b}=\lim _{n \rightarrow+\infty}\left(J_{a b}^{(n)} \times\left(J_{a b}^{(n)}\right)^{T}\right)^{1 / 2 n}
$$

where by $J_{a b}^{(n)}$ one denotes the Jacobian matrix of the $n$th iterate of $\mathcal{L}_{a b}$.

Definition 2.1. Denoting by $\left(\Lambda_{a b}\right)_{i}$ the eigenvalues of the matrix $M_{a b}$, we have that the Lyapunov exponents $\left(\lambda_{a b}\right)_{1}<\left(\lambda_{a b}\right)_{2}$, associated with the Lozi attractor $\mathbf{A}_{\mathcal{L}_{a b}}$, are given by

$$
\left(\lambda_{a b}\right)_{i}=\log \left(\Lambda_{a b}\right)_{i},
$$

with $i=1,2$.

Then, we have the following result.

Theorem 2.2. Given a Lozi map $\mathcal{L}_{a b}$, the largest Lyapunov exponent $\lambda=\left(\lambda_{a b}\right)_{2}$, associated with its attractor $\mathbf{A}_{\mathcal{L}_{a b}}$, is given by

$$
\lambda=\lim _{n \rightarrow+\infty} \frac{1}{2 n} \log \left(\left(A_{n}\right)^{2}+\left(A_{n+1}\right)^{2}+\left(B_{n}\right)^{2}+\left(B_{n+1}\right)^{2}\right),
$$

with

and

$$
\left\{\begin{array}{l}
A_{0}=0 \\
A_{1}=b \\
A_{n+1}=\left(-\varepsilon_{n} a\right) A_{n}+b A_{n-1}
\end{array}\right.
$$

$$
\left\{\begin{array}{l}
B_{0}=1, \\
B_{1}=-\varepsilon_{0} a, \\
B_{n+1}=\left(-\varepsilon_{n} a\right) B_{n}+b B_{n-1} .
\end{array}\right.
$$


Proof. Let

$$
T(x)=\left[\begin{array}{cc}
\mp a & 1 \\
b & 0
\end{array}\right]\left(\frac{1}{x}\right)=\frac{\mp a+x}{b}
$$

be a linear fractional transformation and let $G(x)=1 / T(x)$. Then, the matrix $J_{a b}^{(n)}=J_{a b}^{(n-1)} J_{a b}^{(n-2)} \cdots J_{a b}^{(0)}$ is given by the $n$th composition $G(G(G(\cdots G(x))))$.

Next, let us consider the continuous fraction defined by $G(G(G(\cdots G(x))))$. If one denotes the successive numerators and denominators of the fraction by $A_{n}$ and $B_{n}$, respectively, then the fundamental recurrence formulas are given by

and

$$
\left\{\begin{array}{l}
A_{0}=0, \\
A_{1}=b, \\
A_{n+1}=\left(-\varepsilon_{n} a\right) A_{n}+b A_{n-1}
\end{array}\right.
$$

Therefore,

$$
\left\{\begin{array}{l}
B_{0}=1, \\
B_{1}=-\varepsilon_{0} a, \\
B_{n+1}=\left(-\varepsilon_{n} a\right) B_{n}+b B_{n-1} .
\end{array}\right.
$$

and the eigenvalues of $M_{a b}$ verify, for $i=1,2$,

$$
J_{a b}^{(n-1)} J_{a b}^{(n-2)} \cdots J_{a b}^{(0)}=\left[\begin{array}{ll}
B_{n+1} & B_{n} \\
A_{n+1} & A_{n}
\end{array}\right]
$$

$$
\left(\Lambda_{a b}\right)_{i}^{2}-\left(\Lambda_{a b}\right)_{i} \operatorname{tr} M_{a b}+\operatorname{det} M_{a b}=0,
$$

where

$$
\operatorname{tr} M_{a b}=\lim _{n \rightarrow+\infty}\left(A_{n}\right)^{2}+\left(A_{n+1}\right)^{2}+\left(B_{n}\right)^{2}+\left(B_{n+1}\right)^{2} .
$$

Since we assumed that $0<b<1$, we have $\operatorname{det} M_{a b}=0$ and the result follows immediately.

With this result, we claim that the largest Lyapounov exponent associated with the attractor $\mathbf{A}_{\mathcal{L}_{a b}}$ can be computed solely from the parameters $(a, b)$ and the forward itinerary of a point belonging to the attractor. Having this in mind, next we will compute the Lyapounov exponent $\lambda_{a b}$ for a certain line of the parameter region $\triangle_{\mathcal{L}}$, for which the forward itinerary of the point $I$ is known.

Theorem 2.3. Consider a Lozi map $\mathcal{L}_{a b}$ with parameter values $(a, b)$ from the right boundary of $\triangle_{\mathcal{L}}$, i.e., the line $b=4-2 a$. Then, the largest Lyapunov exponent associated with the attractor $\mathbf{A}_{\mathcal{L}_{a b}}$ is given by

$$
\lambda_{a b}=\log (2) .
$$

Proof. As we know, for parameter values $(a, b)$ belonging to the boundary line $b=4-2 a$, the forward itinerary of the point $I$ is the symbolic sequence $+1-1^{\infty}$. Thus, from (5), we have that, for $n \geq 2$,

$$
\frac{A_{n-1}}{A_{n}}=-\frac{a}{b}+\frac{\frac{1}{b}}{-\frac{a}{b}+\frac{\frac{1}{b}}{-\frac{a}{b}+\ddots}}
$$

As one can easily see, this continued fraction expansion is the solution of the second order equation $b x^{2}+a x-1=$ 0 given by

$$
x=\frac{-a+\sqrt{a^{2}+4 b}}{2 b} .
$$

Thus, for $(a, b)$ belonging to the line $b=4-2 a$, we have $x=\frac{1}{2}$, and

$$
A_{n}=2 A_{n-1} .
$$


In a similar way, we can conclude that $B_{n}=2 B_{n-1}$, for $n \geq 1$. Therefore,

$$
\begin{aligned}
\left(A_{n}\right)^{2}+\left(A_{n+1}\right)^{2}+\left(B_{n}\right)^{2}+\left(B_{n+1}\right)^{2} & =5 \times 2^{2 n}\left(\frac{b}{2}\right)^{2}+5 \times 2^{2 n}= \\
& =\left(5(2-a)^{2}+5\right) 2^{2 n}
\end{aligned}
$$

and, finally,

$$
\lambda_{a b}=\lim _{n \rightarrow+\infty} \frac{1}{2 n}\left(\log \left(\left(5(2-a)^{2}+5\right)\right)+2 n \log (2)\right)=\log (2)
$$

The authors would like to thank the anonymous referees for their careful reading and valuable comments which led to an improvement of the paper.

This work has been partially supported by the research center CIMA-UE, FCT-Portugal funding program.

\section{REFERENCES}

[1] Baptista, D., Severino, R., and Vinagre, S., Kneading curves for Lozi maps, Iteration Theory (ECIT '08), GrazerMath. Ber., 354 (2009) 6-14.

[2] Baptista, D., Vinagre, S., and Severino, R., The basin of attraction of Lozi mappings, Int. J. Bifur. Chaos Appl. Sci. Engrg. 19 (3) (2009) 1043-1049.

[3] Ishii, Y., Towards a kneading theory for Lozi mappings I: A solution of pruning front conjecture and the first tangency problem, Nonlinearity 10, 731-747, 1997.

[4] Ishii, Y. and Sands, D., Monotonicity of the Lozi Family Near the Tent-Maps, Commun. Math. Phys., 198, 397-406, 1998.

[5] Lozi, R., Un ettracteur étrange du type attracteur de Hénon, J. Physique (Paris) 39 (Coll. C5) 9-10, 1978.

[6] Liu, Z., Xie, H., Zhu, Z., and Lu, Q. The strange attractor of the Lozi mapping, Int. J. Bifur. Chaos Appl. Sci. Engrg. 2(4) (1992) 831-839.

[7] Oseledets, V. I., Multiplicative ergodic theorem: Characteristic Lyapunov exponents of dynamical systems, Trudy MMO 19, 179-210, 1968. 